\title{
CARTOGRAPHIE DU CHÂTAIGNIER EN ALSACE PAR IMAGERIE SATELLITE MULTI-DATE
}

\author{
Colette Meyer ${ }^{1}$, Mathilde Caspard ${ }^{1}$, Stephen Clandillon ${ }^{1}$, Henri Giraud ${ }^{1}$, Paul de Fraipont ${ }^{1}$, Maren Baumeister ${ }^{2}$ \\ 1: SERTIT, Strasbourg, colette.meyer@sertit.u-strasbg.fr, mathilde.caspard@sertit.u-strasbg.fr \\ 2: CRPF Lorraine-Alsace, maren.baumeister@crpf.fr
}

\begin{abstract}
Résumé
Parmi toutes les essences qui peuplent la forêt alsacienne, le châtaignier est considéré comme un arbre d'avenir face aux enjeux du réchauffement climatique. Cette essence a fait l'objet d'une étude particulière dans le cadre d'un projet franco-allemand intégré au programme européen Interreg IV A Rhin supérieur. D'une durée de trois ans, cette étude avait pour objectif de développer des stratégies de préservation et de valorisation de ces châtaigneraies. Elle a ainsi porté sur la conservation des paysages forestiers, sur la valorisation du bois et des fruits, ainsi que sur la préservation des châtaigneraies face au chancre qui menace les peuplements.

Dans le cadre de ce programme, le SERTIT a développé une méthode pour produire une cartographie détaillée et à jour des zones de châtaigniers sur toute l'Alsace. La méthodologie pour distinguer les châtaigniers des autres essences forestières a été développée à partir d'un choix ciblé d'images satellites acquises à différentes saisons et des données de terrain de placettes géo localisant des châtaigneraies. Ainsi des images SPOT5 et ALOS AVNIR-2 multi-temporelles couvrant différentes phases du cycle de développement annuel du châtaignier (au printemps et en été) ont été utilisées. L'étude est basée sur une analyse des caractéristiques spectrales pour les principaux feuillus à partir d'échantillons de terrain pour les différentes images. Ainsi, l'analyse des signatures spectrales a permis d'identifier les images permettant une différenciation des châtaigniers, ce sont celles acquises au début du printemps et en été. Elles correspondent aux caractéristiques particulières propres à cette essence qui sont son développement foliaire tardif par rapport aux autres feuillus et sa floraison abondante. Les résultats de cette cartographie générée sur l'ensemble de la région Alsace ont été validés lors de campagnes de terrain, cette validation qui n'a été que partielle a pourtant montré une bonne précision de détection des peuplements de châtaigniers matures qui est de $85 \%$.
\end{abstract}

Mots-clés : espèce forestière, châtaigniers, signatures spectrales, images optiques, satellite, haute résolution, série temporelle, série multi saisonnière

\begin{abstract}
Of the many tree species that grow in Alsace forest, chestnut trees are considered as promising and adapted to the challenge of global warning. This species has been the focus of a joint French-German Interreg IV study. This three-year study's objective was to develop conservation strategies and to valorize the sweet chestnut. Therefore, it has focused on conservation, on wood and fruit use, and on sweet chestnut preservation as they are faced a canker disease that threatens the forest stands.

Within this project, a method has been developed for the precise and up-to-date mapping of chestnut stands throughout Alsace. To distinguish chestnut from other species, the method is based on a targeted selection of satellite images acquired at different seasons and field plot data geo-locating chestnut trees. Thus, SPOT 5 and ALOS AVNIR-2 multi temporal images covering the chestnut tree vegetative phases were used. The study is based on the multi-temporal spectral characteristic analysis of the main deciduous species using field samples. In this way, the spectral signature analysis identified images acquired in early spring and in summer as differentiating chestnut trees. They correspond to the particular characteristics of this species which are its late bud break compared to other species and its abundant flowering. The results of this mapping covering the whole of the Alsace region were validated during field campaigns. This partial validation has shown an accuracy of detection concerning mature chestnut stands of $85 \%$.
\end{abstract}

Keywords: forest tree species, chestnut, spectral signature, optical imagery, satellite, high resolution, temporal series, multi-season series

\section{Introduction}

Patrimoine écologique et culturel commun à la Rhénanie Palatinat, au Bade-Wurtemberg et à l'Alsace, la châtaigneraie occupe une place importante dans la frange collinéenne surplombant les vignobles de ces trois régions. Introduit par les romains il y a plus de 2000 ans, le châtaignier a fourni pendant des siècles des piquets de vignes et des feuillards pour encercler les barriques. Malgré ses nombreux atouts pour l'économie régionale et le tourisme, le châtaignier est peu valorisé. Phénomène aggravant, en particulier en Allemagne, il est victime du chancre du châtaignier, champignon responsable de dépérissements inquiétants. Ces éléments ont eu pour conséquence la mise en œuvre d'une étude transfrontalière dans le cadre du programme Interreg IV Rhin supérieur : «Le châtaignier dans la région du Rhin supérieur - une essence rassemblant hommes, cultures et paysages ». 
Ce projet, cofinancé par la Région Alsace, a visé l'élaboration de stratégies pour la sauvegarde et la valorisation de ce patrimoine culturel unique. L'utilisation du bois de châtaignier en viticulture est abandonnée depuis longtemps entrainant une sousexploitation ou même un abandon des taillis en Alsace pour lesquels l'usage du châtaignier reste souvent cantonné à celui du bois de chauffage. Le bois de châtaignier est particulièrement souple et durable, résistant aux insectes et aux intempéries et est comparable à certains bois exotiques. Depuis plusieurs années déjà, des forestiers allemands ont orienté la sylviculture du châtaignier vers la production de bois d'œuvre de qualité. Pourtant, des tiges de qualité menuiserie, charpente, ou paravalanche, sont abondantes dans les taillis alsacien mais les volumes commercialisés sont très faibles. Le projet a permis la publication d'un guide de sylviculture (Baumeister, 2013) afin de promouvoir le châtaignier et son bois et un marché du bois de châtaignier est en train d'émerger en Alsace. En plus, le châtaignier est considéré comme un arbre d'avenir face aux enjeux du réchauffement climatique. En effet les prévisions climatiques pour le XXlème siècle montrent pour l'Alsace une progression probable des espèces caractéristiques de l'Ouest de la France dont le châtaignier fait partie et une vulnérabilité face au changement climatique du hêtre très présent dans la région (Timina, 2012).

La plupart des châtaigneraies en Alsace font partie des petites propriétés de la forêt privée, la localisation de ces châtaigneraies n'était pas toujours bien connue. En effet, les cartes forestières IFN présentent bien cette essence mais ne font ressortir que quelques grandes zones de châtaigniers purs (surface totale de 950 ha pour les cartes IFN V2 sur l'ensemble de l'Alsace). Ainsi, dans le cadre de ce projet, le SERTIT, Service Régional de Traitement d'Image de Télédétection, a été chargé de produire une carte de localisation des zones de châtaignier sur toute l'Alsace à partir d'images satellites. En effet, connaître exactement leur situation permettra de préserver et de valoriser cette essence structurant le paysage des collines sous- vosgiennes.

Bien que technologie récente, les méthodologies de la télédétection constituent déjà un outil fiable, performant, complémentaire des méthodes traditionnellement utilisées pour la gestion et le suivi des forêts. Les données de la télédétection sont utilisées depuis très longtemps pour les inventaires forestiers. Depuis 1990, l'Inventaire forestier national utilise les images satellitaires à résolution décamétrique pour fournir à ses utilisateurs une information sur la forêt. Depuis cette époque, l'usage de la télédétection permet de réaliser le suivi du couvert forestier par la détection des coupes forestières, et, dans les années 2000, la cartographie de dégâts des tempêtes (IFN, 2008). Les informations de la ressource forestière que l'on cherche à obtenir à partir des données de la télédétection sont variées, il peut s'agir de la structure de la forêt, de son état sanitaire, des changements de surface ou des essences. Dans de nombreuses applications pratiques, l'interprétation visuelle des images est encore utilisée pour extraire les informations nécessaires. Mais, elle est d'un coût élevé et ses résultats dépendent beaucoup des photo-interprètes (Koch, 2012).

La séparabilité feuillus / conifères à partir d'images optiques a été montrée dès les années 1980 mais la différenciation des différentes espèces est plus problématique à cause des caractéristiques spectrales pouvant être similaires (Weigel et al, 2012). De nombreuses études visent à identifier et à cartographier les différentes essences forestières. Des approches au niveau du pixel sont encore utilisées avec des images à haute résolution spatiale pour discriminer ces essences (Mafhoud, 2010; Mahboob et al, 2011). Beaucoup d'études portent sur des images à très haute résolution et utilisent des classifications basées sur des approches « orientées objets " (Hàjek, 2005). L'utilisation de la télédétection se développe également grâce aux avancées technologiques et plus particulièrement dans le domaine des méthodes de classification (Wang et al, 2010). En classification supervisée, de nombreuses méthodes ont été utilisées pour cartographier la couverture forestière. On peut citer l'algorithme de classification "Random forest " qui, à l'échelle de l'arbre, a permis d'identifier 10 essences avec une approche objet à partir d'une image très haute résolution WordView-2 (Immitzer et al, 2012).

Les méthodes citées ci-dessus n'utilisent souvent qu'une seule image. II a été montré que l'utilisation de séries temporelles de données à basse résolution (par ex: Modis, Spot Vegetation) permettent le suivi du cycle phénologique des essences (Guyon et al. 2011) et ces images sont de plus en plus utilisées pour les analyses environnementales. Toutefois la résolution spatiale de ces données ne permet qu'un suivi de la végétation forestière dans son ensemble.

Notre étude a utilisé des données décamétriques à haute résolution. À partir de relevés de terrain géolocalisant des châtaigniers, fournis par la CRPF (Centre Régional de la Propriété Forestière) de Lorraine-Alsace, et d'un choix ciblé d'images satellites acquises à différentes saisons, une méthode permettant de différencier le châtaignier des autres essences forestières a été développée. La méthodologie, appliquée sur plusieurs zones test réparties sur l'Alsace et validée par des campagnes de terrain effectuées par le CRPF, a permis d'obtenir une cartographie précise des châtaigneraies en Alsace.

\section{Site d'étude et données utilisées}

Le site d'étude couvre l'ensemble de l'Alsace. En effet, le châtaignier caractérise le paysage des collines en bordure du massif vosgien au-dessus des vignobles. 
Des images satellites multispectrales SPOT 5 (résolution de $10 \mathrm{~m}$ ) acquises à différentes périodes de l'année ont été utilisées pour l'étude. Ne disposant pas d'assez de données d'une même année, les images utilisées ont été acquises sur plusieurs années d'avril à septembre, afin de bien couvrir tout le cycle de développement annuel du châtaignier (Tableau 1). L'image acquise le 5 juillet ne couvrant pas l'ensemble du secteur d'étude a été complémentée par une image multispectrale également d'une résolution de $10 \mathrm{~m}$ issues du capteur ALOS AVNIR-2 et acquise le 17 juin 2009. Les caractéristiques des différentes images sont données dans les tableaux 2 et 3 .

\begin{tabular}{|c|c|c|}
\hline Date d'acquisition & Type d'image & Secteur \\
\hline 7 avril 2010 & $\begin{array}{c}\text { SPOT } 5 \\
\text { multispectrale }\end{array}$ & Tout \\
\hline 25 avril 2007 & $\begin{array}{c}\text { SPOT } 5 \\
\text { multispectrale }\end{array}$ & Tout \\
\hline 17 juin 2009 & $\begin{array}{c}\text { ALOS } \\
\text { AVNIR-2 }\end{array}$ & Tout \\
\hline 5 juillet 2010 & $\begin{array}{c}\text { SPOT } 5 \\
\text { multispectrale }\end{array}$ & $\begin{array}{l}3 / 4 \text { de la zone } \\
\text { (extrêmes nord et sud } \\
\text { manquants) }\end{array}$ \\
\hline 20 août 2009 & $\begin{array}{c}\text { SPOT } 5 \\
\text { multispectrale }\end{array}$ & Tout \\
\hline 13 septembre 2007 & $\begin{array}{c}\text { SPOT } 5 \\
\text { multispectrale }\end{array}$ & $2 / 3$ est de la zone \\
\hline
\end{tabular}

Tableau 1 : Images utilisées pour l'étude

\begin{tabular}{|c|c|c|c|c|}
\hline $\begin{array}{c}\mathbf{N}^{\circ} \text { de } \\
\text { bande }\end{array}$ & $\begin{array}{c}\text { Domaine } \\
\text { spectral }\end{array}$ & $\begin{array}{c}\text { Longueur } \\
\text { d'onde } \\
\text { (en } \mathbf{\mu m})\end{array}$ & $\begin{array}{c}\text { Taille de } \\
\text { la }\end{array}$ \\
\hline (en m)
\end{tabular}

Tableau 2 : Caractéristiques des images multi-spectrales issues du satellite SPOT 5

\begin{tabular}{|c|c|c|c|c|}
\hline $\begin{array}{l}\mathrm{N}^{\circ} \text { de } \\
\text { bande }\end{array}$ & $\begin{array}{l}\text { Domaine } \\
\text { spectral }\end{array}$ & $\begin{array}{l}\text { Longueur } \\
\text { d'onde } \\
\text { (en } \mu \mathrm{m})\end{array}$ & $\begin{array}{l}\text { Résolution } \\
\text { (en } \mathrm{m})\end{array}$ & $\begin{array}{c}\text { Taille de } \\
\text { la } \\
\text { fauchée } \\
\text { (en } \mathrm{km} \text { ) }\end{array}$ \\
\hline MS1 & Bleu (B) & $0.42-0.50$ & \multirow{4}{*}{10} & \multirow{4}{*}{$70 \times 70$} \\
\hline MS2 & Vert (V) & $0.52-0.60$ & & \\
\hline MS3 & Rouge (R) & $0.61-0.69$ & & \\
\hline MS4 & $\begin{array}{l}\text { Proche } \\
\text { Infrarouge } \\
\text { (PIR) }\end{array}$ & $0.76-0.89$ & & \\
\hline
\end{tabular}

Tableau 3 : Caractéristiques des images issues du capteur ALOS AVNIR-2
Les images satellites sont reçues dans un format brut et ont été orthorectifiées, en utilisant comme référence une image SPOT 5 de 2002 orthorectifiée par l'IGN et une vingtaine de points saisis sur cette image, afin de les intégrer dans la base de données. Des corrections exo-atmosphériques (réflectance au-dessus de l'atmosphère TOA) ont également été appliquées aux images avec des données finales en comptes numériques.

Des châtaigneraies ont pu être localisées grâce, d'une part à une cinquantaine de placettes (d'une quinzaine de mètres de rayon) de châtaigniers purs relevées sur le terrain en 2011 par le CRPF et, d'autre part, à des données de terrain sur des parcelles forestières gérées par la forêt privée (en provenance du CRPF et de coopératives forestières privées) (Figure 1).

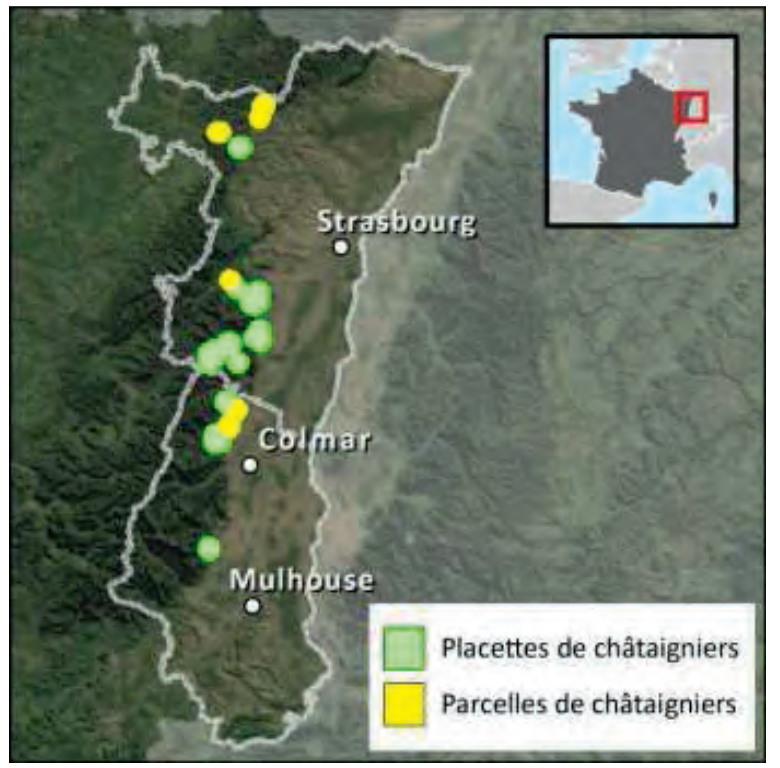

Figure 1 : Localisation des placettes et parcelles de châtaignier utilisées pour développer la méthodologie

Les données de gestion sur les parcelles, contenant des informations relatives aux essences, structure, typologie et classe d'âge, relevées entre 2004 et 2009, ont permis de repérer des échantillons des autres espèces de feuillus. Des parcelles mono-spécifiques ont été sélectionnées pour les principaux feuillus présents dans le massif vosgien, qui sont le hêtre et le chêne. Ainsi une cinquantaine de parcelles de quelques dizaines d'ares chacune ont été utilisées pour chaque type de feuillus.

\section{Démarche méthodologique}

L'étude s'appuie sur le calendrier végétatif particulier du châtaignier par rapport aux autres feuillus : son développement foliaire tardif et sa floraison abondante (Figure 2). 


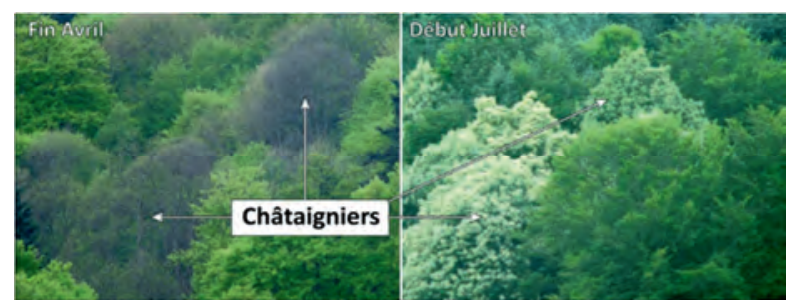

Figure 2 : Photographies illustrant le développement foliaire tardif et la floraison des châtaigniers, avec à gauche, cliché pris fin avril, à droite cliche pris début juillet

La sortie des feuilles pour le châtaignier se fait tardivement, généralement à la fin du mois d'avril, bien après celui des autres feuillus tels que le hêtre ou le chêne qui sont les feuillus prépondérants en Alsace. Lors de sa floraison fin juin qui dure quinze jours il produit une quantité importante de grandes fleurs blanc cassé (Figure 3), ce qui permet de le distinguer aisément des autres essences forestières dans le paysage (Figure 4).

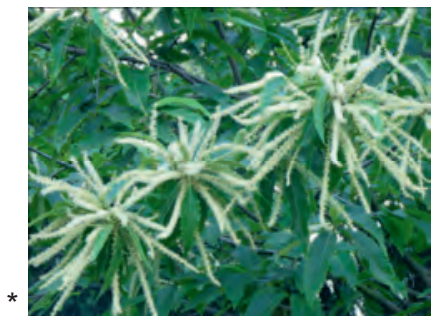

Figure 3 : Floraison du châtaignier

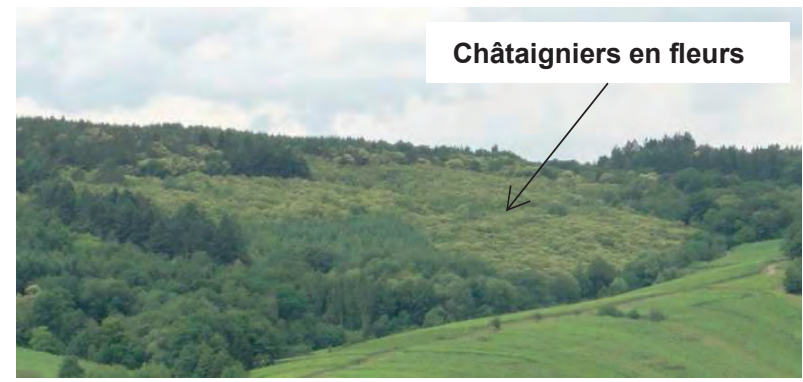

Figure 4 : Châtaigniers en fleur dans le paysage

L'étude est basée sur une analyse comparée des caractéristiques spectrales des trois espèces de feuillus à partir des échantillons de peuplement pour les différentes images et pour toutes les bandes spectrales. Cette analyse permet d'identifier les images et les canaux qui seront utilisés pour la cartographie des châtaigniers,

\subsection{Analyse des caractéristiques spectrales}

Les figures 5 à 7 présentent les résultats intéressants pour le canal rouge sur la zone test de Villé (Figure 9). Des parcelles de châtaignier, de hêtre et de chêne, un nombre de 11,12 et 13 respectivement, représentant respectivement une surface de 15 ha, 18 ha et 56 ha, ont été utilisées.

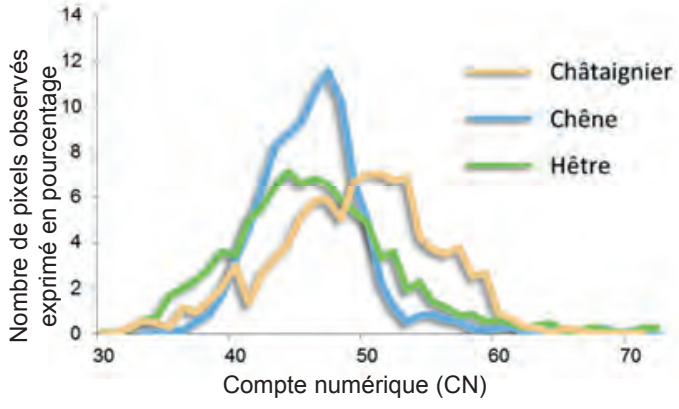

Figure 5 : Signatures spectrales du châtaignier, du hêtre et du chêne dans le canal rouge pour l'image SPOT5 acquise le 25 avril 2007

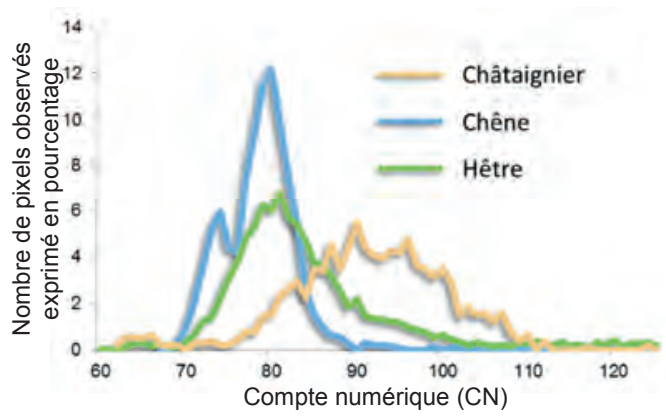

Figure 6 : Signatures spectrales du châtaignier, du hêtre et du chêne dans le canal rouge pour l'image SPOT5 acquise le 5 juillet 2010

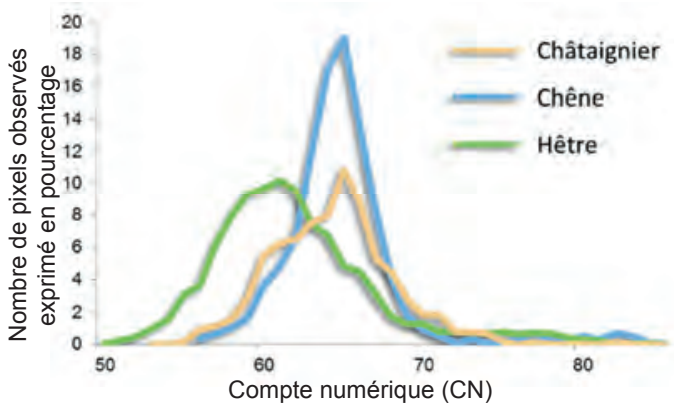

Figure 7 : Signatures spectrales du châtaignier, du hêtre et du chêne dans le canal rouge pour l'image SPOT5 acquise le 20 août 2009

L'analyse des signatures spectrales montre que les châtaigniers se différencient des autres feuillus en avril (développement foliaire tardif) et en juillet lors de leur floraison. Ce sont donc ces deux images qui ont été utilisées pour les différentier des autres feuillus.

C'est le canal rouge qui permet de différencier au mieux les châtaigniers mais la distinction est également perceptible pour le canal vert, ces deux canaux ont dont été utilisés pour leur cartographie.

\subsection{Cartographie des châtaigniers}

Pour la cartographie des châtaigniers le logiciel ERDAS Imagine a été utilisé. Plusieurs étapes basées sur des classifications supervisées pixel à pixel ont été nécessaires. Les traitements consistent en différentes 
étapes de seuillages (classification par hypercubes) de différents canaux et de l'Indice de Végétation Normalisée (IVN) suivis pour chaque étape de traitements de nettoyage et de lissage des couches obtenues.

Les différentes étapes, illustrées sur un extrait par la figure 8, sont :

- l'extraction du masque forestier à partir de l'image acquise le 20 août 2009 (utilisation des canaux vert, rouge, PIR, IVN),

- à l'intérieur du masque forestier, une différenciation entre les feuillus et les conifères grâce à l'image du 7 avril 2010 acquise avant la sortie des feuilles des arbres caduques (utilisation des canaux rouge, PIR, IVN),

- à l'intérieur des secteurs classés comme feuillus, l'identification des châtaigniers par une classification par hypercubes dans les canaux vert et rouge des deux images d'avril et de juillet. Les seuils ont été définis en se basant sur les signatures spectrales réalisées précédemment (cf 3.1). L'image classée, qui est "bruitée", est lissée à l'aide d'un filtrage d'image, en utilisant des filtres de dilatation et d'érosion de la morphologie mathématique, puis nettoyée en éliminant les petits objets de moins de 10 ares.

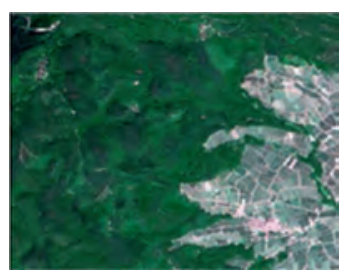

$\stackrel{0}{0} \mathrm{~km}$

Image SPOT 5 acquise le 20 août 2009

Différentiation

Feuillus/Conifères

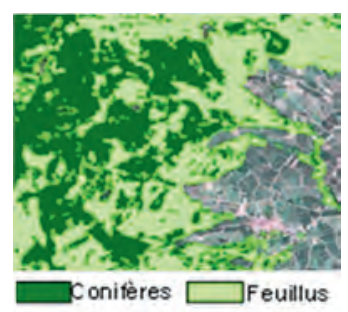

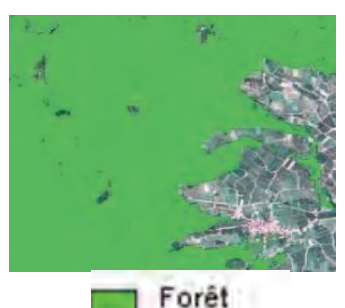

Forêt

Extraction du masque forêt

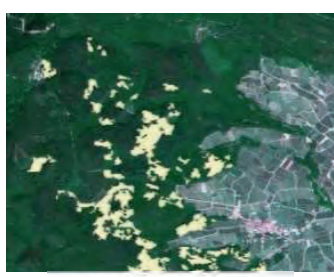

$\square$ Chầtaignier

Identification des Châtaigniers
Figure 8 : Exemple d'image utilisée et résultats des différentes étapes de la cartographie des châtaigniers

\section{Résultats et discussion}

La méthodologie mise au point a d'abord été appliquée sur cinq zones test d'une surface de quelques dizaines de $\mathrm{km}^{2}$ réparties sur l'ensemble du massif vosgien alsacien (Figure 9).

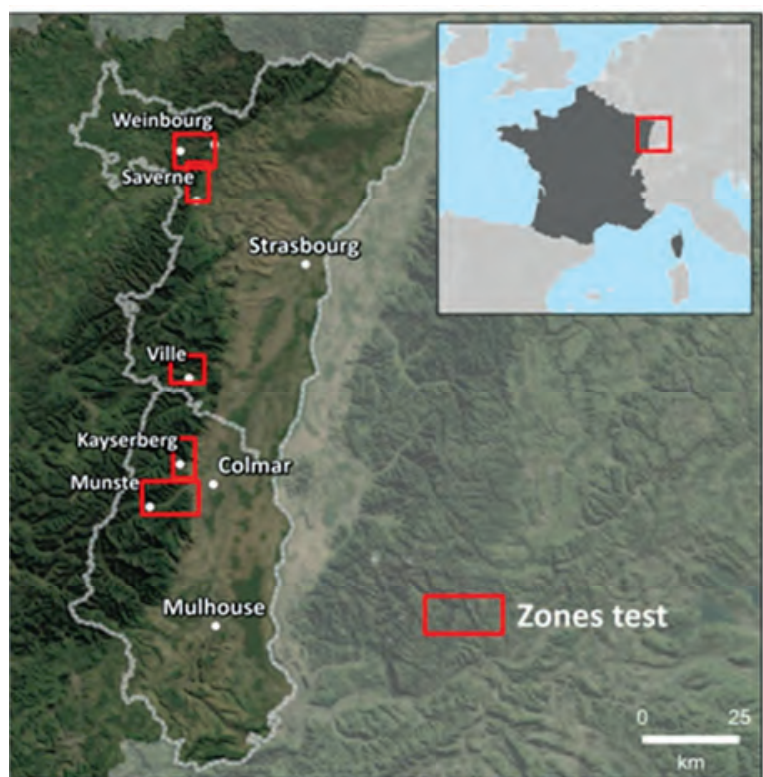

Figure 9 : Localisation des zones test en Alsace

Pour chaque zone test une carte des châtaigneraies de plus de 10 ares a été produite à partir des images satellite multi-dates (Figure 10).

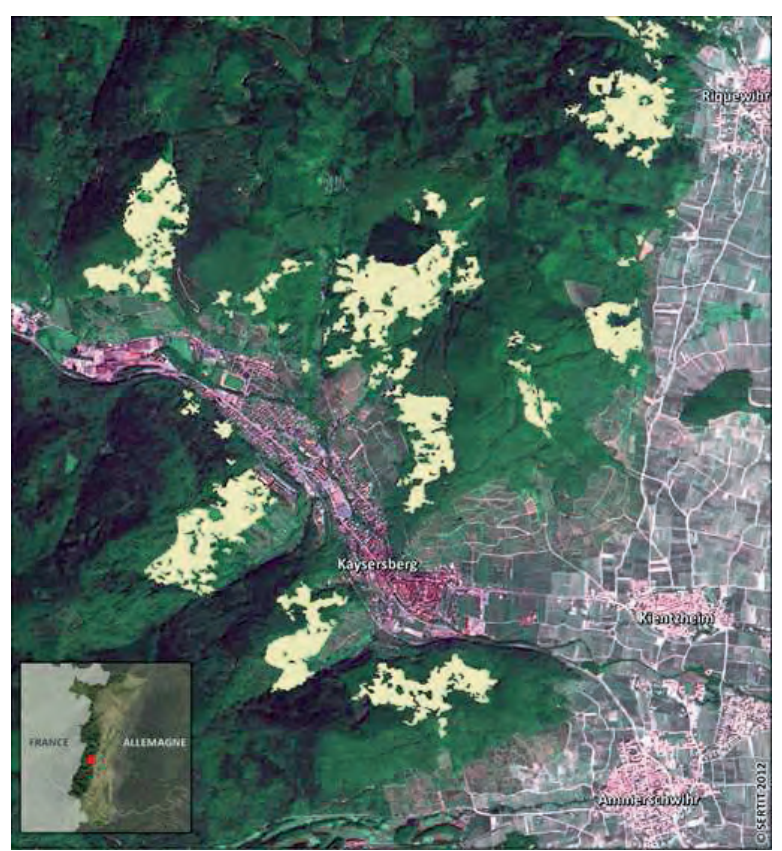

Figure 10 : Carte des châtaigneraies produite pour la zone test de Kaysersberg

Les cartes obtenues ont fait l'objet d'une validation sur le terrain. Une sélection aléatoire, d'une part de points situés dans les zones de châtaigneraies cartographiées, et d'autre part de points classés non châtaigniers localisés dans des secteurs de feuillus proches des châtaigneraies, a été faite par le SERTIT (Figure 11). La validation réalisée n'a été que partielle car elle n'a été faite que dans les châtaigneraies et leur bordure. Lors des campagnes de terrain, les agents du 
CRPF ont visité les points sélectionnés en se repérant à l'aide d'un GPS. Ils ont relevé pour chaque point une estimation " à dire d'expert " de la proportion du couvert en châtaigniers (en classe de 10\%) et une description de leur typologie (taillis ou futaie). Les estimations du taux de couvert ont ensuite été regroupées en classes de $25 \%$.

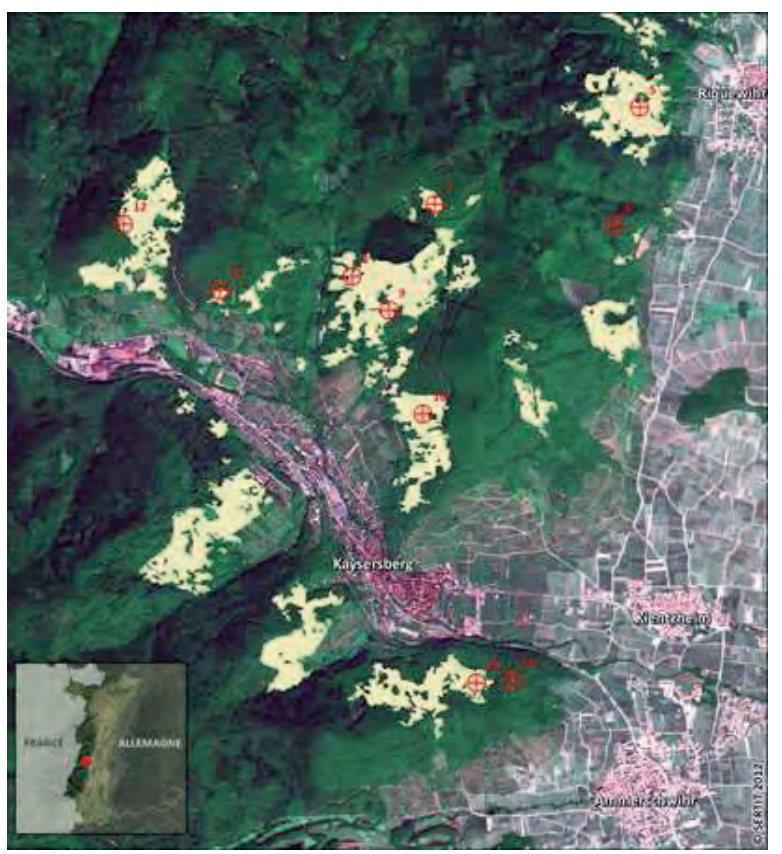

Figure 11 : Sélection de points pour la validation de la zone test de Kaysersberg

Pour l'ensemble des zones test, 71 points ont ainsi été contrôlés (Tableau 4).

\begin{tabular}{|c|c|c|c|}
\hline & \multicolumn{2}{|c|}{ Classification } & \\
\hline $\begin{array}{c}\text { Relevés } \\
\text { terrain }\end{array}$ & Châtaigniers & $\begin{array}{c}\text { Non châtaigniers } \\
\text { en bordure des } \\
\text { châtaigneraies }\end{array}$ & Total \\
\hline Châtaigniers & $\mathbf{5 0}$ & $\mathbf{9}$ & 59 \\
\hline $\begin{array}{c}\text { Non } \\
\text { châtaigniers }\end{array}$ & $\mathbf{2}$ & $\mathbf{1 0}$ & 12 \\
\hline Total & 52 & 19 & 71 \\
\hline
\end{tabular}

Tableau 4 : Validation sur le terrain de la classification

L'estimation de la qualité de la classification peut en être déduite, ainsi, le taux de pixels bien classés est de $85 \%$. Les taux d'erreur et d'omission sont respectivement de $3 \%$ et de $13 \%$ pour la classe châtaignier seulement.

Les 52 points contrôlés dans les châtaigneraies cartographiées ont mis en évidence la présence de châtaigniers pour $96 \%$ des points montrant une très bonne détection des châtaigniers et validant ainsi la méthodologie.

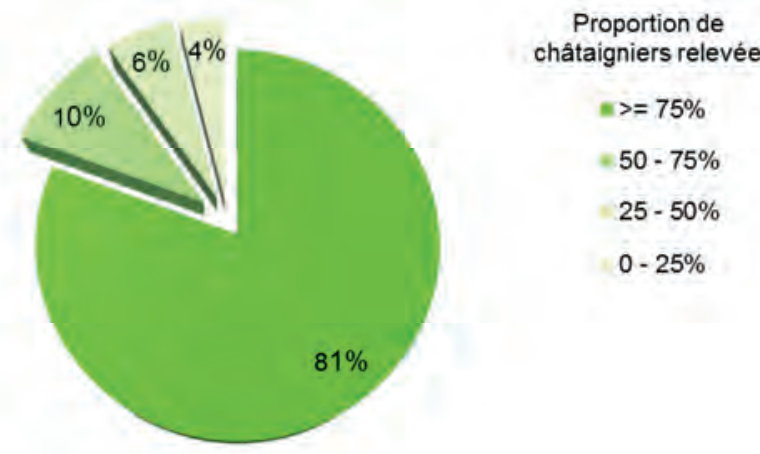

Figure 12 : Résultats concernant les points contrôlés dans les zones classées comme châtaigneraies

En ce qui concerne les 19 points sélectionnés dans des secteurs proches des châtaigneraies et classés non châtaigniers, l'absence de châtaigniers a été confirmée pour 10 points. Toutefois la présence de jeunes taillis de châtaigniers a été mise en évidence pour quelques points visités, ceci explique le taux d'omission plutôt élevé. Comme la méthodologie s'appuie sur la floraison des châtaigniers, elle ne permet pas toujours de détecter les jeunes châtaigneraies si les arbres ne fleurissent pas.

Cette validation partielle n'a pas permis d'évaluer la précision de la classification pour la classe «non châtaigniers » à cause de la sélection non aléatoire sur l'ensemble de la zone d'étude.

La cartographie obtenue a été comparée à la carte IFN V2. Cette dernière ne fait ressortir que quelques grandes zones de châtaigneraies, toutes ces grandes zones ont bien été mises en évidence sur la cartographie. Par contre la cartographie a permis de repérer les châtaigneraies situées en bordure du massif Vosgiens juste au-dessus du vignoble, bien visibles dans le paysage lors de leur floraison, non présentes sur carte IFN V2.

Les campagnes de terrain ont montré une bonne détection des peuplements de châtaigniers matures validant partiellement la méthodologie. La méthodologie élaborée a donc été généralisée et a ainsi permis d'obtenir une cartographie des grandes zones de châtaigneraies matures sur l'ensemble de la région Alsace (Figure 13). Les châtaigneraies, d'une superficie totale estimée à partir de cette cartographie à près de 2 500 ha, sont essentiellement localisées le long du vignoble en bordure du massif Vosgien mais aussi dans le Parc Naturel Régional des Vosges du Nord, les plus grandes châtaigneraies étant situées dans le centreAlsace. 


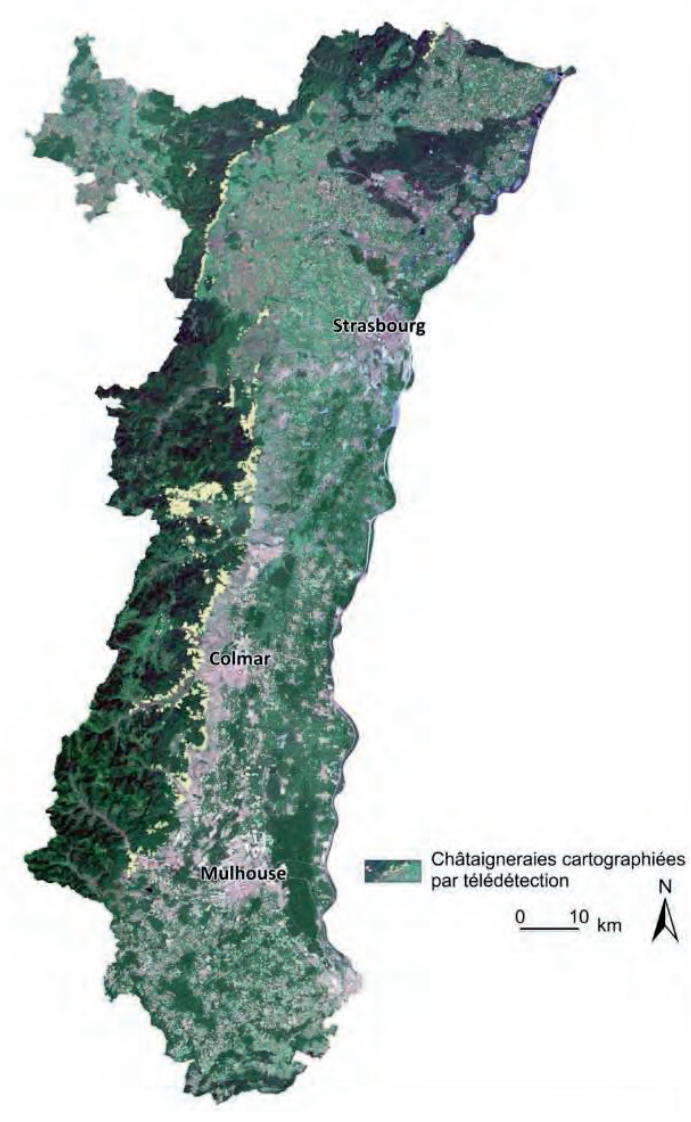

Figure 13 : Cartographie des châtaigniers sur l'ensemble de l'Alsace

\section{Conclusion et perspectives}

A partir d'images satellite multi-temporelles optiques et de résolution décamétrique, couvrant différentes phases du cycle de développement annuel du châtaignier et de données de terrain géo-localisant des châtaigneraies, une méthodologie permettant sa différenciation des autres essences forestières et sa cartographie a ainsi été mise au point en collaboration avec le CRPF de Lorraine-Alsace. La méthodologie évaluée à partir de relevés sur le terrain a donné de très bons résultats pour les peuplements de châtaigneraies matures.

La méthodologie originale, basée sur l'utilisation d'images satellites acquises à différentes saisons, pourrait être appliquée à une autre région afin d'y repérer ses châtaigniers, elle pourrait également être développée pour la cartographie d'autres essences forestières.

L'étude a ainsi montré des résultats prometteurs pour la différentiation des espèces forestières avec des séries temporelles. En effet, les séries temporelles permettent de disposer d'images acquises dans la bonne fenêtre d'observation afin de suivre le développement végétal, qui varie pour les différentes essences mais qui fluctue aussi d'une année à l'autre en fonction des conditions météorologiques. L'exploitation de la série temporelle des futures données Sentinel-2 grâce à ses images à haute résolution acquises à grande fréquence temporelle couvrant la totalité du cycle de développement annuel, permettront encore un meilleur suivi des systèmes forestiers.

\section{Références}

Baummeister M., 2013, Un guide transfrontalier pour la sylviculture du châtaignier, Floréal 94, 6-7.

Guyon D., Guillot M., Vitasse y., Cardot H., Hagolle O., Delzon S., Wigneron J.P., 2011. Monitoring elevation variations in leaf phenology of deciduous broadleaf forests from SPOT/VEGETATION time-series. Remote Sensing of Environment 115, 615-627.

Hájek F., 2005. Object-oriented classification of remote sensing data for the identification of tree species composition. Proc. Conf. ForestSat 2005, Boras, 31. 5. - 3. 6. 2005: $16-20$.

IFN, 2008, les images satellitaires pour la gestion forestière, l'IF $n^{\circ} 18,8 p$.

Immitzer M., Atzberger C, Koukal T., 2012. Tree Species Classification with Random Forest Using Very High Spatial Resolution 8-Band WorldView-2 Satellite Data, Remote Sensing 2012, 4, 2661-2693.

Koch B., 2012, La télédétection et son utilisation dans les évaluations forestières nationales (EFN). Répertoire des connaissances sur évaluations des ressources forestières nationales, catalogue de la FAO, 18p.

Mahboob J., Khattak U., Rahman M., 2011. Forest tree species classification using multispectral satellite imageries. Proceedings GTC 2011, 10-13 May, 2011, Jeddah, KSA.

Mafhoud I., 2009. Cartographie et mesure de la biodiversité du Mont Ventoux Approche par Système d'Information Géographique et Télédétection, préconisations méthodologiques et application pour l'aménagement forestier. Thèse de doctorat de l'Université d'Avignon et des pays de Vaucluse, 191p.

Timina J., Bertin M., 2012, Vulnérabilité des forêts alsaciennes face au changement climatique Rapport DREAL ALSACE - REGION ALSACE, 21p.

Wang K., Franklin S. E., Guo X., Cattet M., 2010, Remote Sensing of Ecology, Biodiversity and Conservation: A Review from the Perspective of Remote Sensing Specialist, Sensors 2010, 10, 96479667.

Weigel D, Randolph J.C., 2012. Spectral reflectance of five hardwood tree species in southern Indiana. Proceedings of the 18th Central Hardwoods Forest Conference, 276-282. 